\title{
1 Optimization of the navigated TMS mapping algorithm 2 for accurate detection of plasticity and abnormalities in 3 cortical muscle representations
}

\author{
Dmitry O. Sinitsyn ${ }^{1 *}$, Andrey Yu. Chernyavskiy ${ }^{1,2}$, Alexandra G. Poydasheva ${ }^{1}$, Ilya S. Bakulin ${ }^{1}$, \\ Natalia A. Suponeva ${ }^{1}$, Michael A. Piradov ${ }^{1}$ \\ 1 Research Center of Neurology, 125367 Moscow, Russia; d_sinitsyn@mail.ru (D.S.); \\ andrey.chernyavskiy@gmail.com (A.C.); alexandra.poydasheva@gmail.com (A.P.); bakulinilya@gmail.com (I.B.); \\ nasu2709@mail.ru (N.S.); mpi711@gmail.com (M.P.) \\ 2 Valiev Institute of Physics and Technology of Russian Academy of Sciences, 117218 Moscow, Russia \\ * Correspondence: d_sinitsyn@mail.ru
}

\begin{abstract}
11 Abstract: Navigated TMS mapping of cortical muscle representations allows noninvasive assessment of the state of a healthy or diseased motor system and monitoring its change with time. These applications are hampered by the heterogeneity of existing mapping algorithms and the lack of detailed information about their accuracy. We aimed to find an optimal motor evoked potential (MEP) sampling scheme in the grid-based mapping algorithm in terms of the accuracy of muscle representation parameters. The APB muscles of eight healthy subjects were mapped three times on consecutive days using a seven-by-seven grid with ten stimuli per cell. The effect of the MEP variability on the parameter accuracy was assessed using bootstrapping. The accuracy of representation parameters increased with the number of stimuli without saturation up to at least ten stimuli per cell. The detailed sampling showed that the between-session representation area changes in the absence of interventions were significantly larger than the within-session fluctuations and thus could not be explained solely by the trial-to-trial variability of MEPs. The results demonstrate that the number of stimuli has no universally optimal value and must be chosen by balancing the accuracy requirements with the mapping time constraints in a given problem.
\end{abstract}

Keywords: navigated transcranial magnetic stimulation; TMS motor mapping; cortical muscle representation; bootstrapping; variability; accuracy

\section{Introduction}

Mapping cortical motor representations of muscles using navigated transcranial magnetic stimulation (nTMS) is a valuable noninvasive method providing information about the motor system that is useful for research and clinical purposes [1-3]. Its ability to localize motor eloquent cortical areas has found successful applications in preoperative planning [4,5]. Additionally, a growing body of literature is concerned with the use of nTMS mapping for assessing the state of the motor system and its plastic changes during learning of new skills [6-9], in neurological diseases, such as stroke [10], dystonia [11], spinal cord injury [12,13], amyotrophic lateral sclerosis [14], as well as in the course of treatment [15]. For identifying the possibly subtle differences in motor maps, it is essential to make the method precise and reliable. Meanwhile, the high variability of motor evoked potentials (MEPs), on which the TMS-maps are based, makes the accurate estimation of representation parameters challenging [16-18].

The interpretation of the results of TMS mapping is complicated by the lack of a standard protocol and the existence of a wide variety of approaches to the mapping procedure, the selection of the studied muscle representation parameters and methods of their calculation [10,19]. One of the most frequently used approaches is based on a predefined grid of cortical points with application of a fixed number of stimuli at each point $[20,21]$. The studies using this method are heterogeneous in terms of the number of grid cells, their size and the number of stimuli per cell [10,21-25]. Given the high variability of MEPs, the number of stimuli per cell is an important factor influencing the accuracy of the representation parameters [20,26].

The reproducibility of muscle representation parameters and their stability in the absence of interventions is one of the key aspects for the application of navigated TMS motor mapping for research and clinical purposes $[16,27]$. The studies conducted to date have obtained divergent results with regard to the intraclass correlation coefficient (ICC) for various parameters of cortical representations, ranging from 0.36 to 0.89 [16,24,27]. A number of approaches to reducing the variability of muscle representation parameters have been proposed, such 
as neuronavigation by an individual structural MRI for improving the repeatability of coil placement and orientation [28,29] and taking into account the individual topography and morphology of the cerebral cortex [30-32]. Another promising research direction is the brain-state dependent stimulation based on combining EEG and TMS in real time to align the stimulus times with EEG features, such as the $\mu$-rhythm phase [33].

A general approach to dealing with the trial-to-trial variability of MEPs is averaging multiple measurements [34,35]. In agreement with probability theory, the accuracy of some muscle representation parameters has been reported to increase with the number of stimuli used during the mapping [20]. However, comprehensive knowledge of this dependence for all the common parameters is lacking, and it is unknown whether the increase in the accuracy saturates (reaches a plateau) after a certain number of stimuli. This is important for estimating the payoff in the quality of the data that a researcher obtains from investing the subject's and operator's time and effort into the detailed mapping of muscle representations.

Another open question regarding the averaging approach is whether it can reduce to an arbitrary degree the session-to-session variability of muscle representation parameters in the absence of interventions. Averaging makes the parameters closer to their exact mean (expected) values in a given session, and these values will not necessarily be the same in a different session. Thus, it is important to test whether the variations of muscle representation parameters between sessions can be fully explained by the trial-to-trial MEP variability within a session and can thus be controlled by sufficient sampling of MEPs. An alternative scenario is the existence of systematic between-session changes of the MEP probability distributions, which cannot be influenced by the sampling scheme.

The existing data analysis methods in TMS mapping differ in their definitions of muscle representation parameters. The area of a representation mapped using the grid-based method has been defined as the total area of the cells with at least one suprathreshold MEP out of three stimuli [22], at least five out of ten [23], six out of ten [21,24], or two out of six [25] suprathreshold MEPs. Several studies have studied the area in which an interpolated mean amplitude function exceeds some threshold, with varying interpolation methods and thresholds [17,36,37]. Recently, a more advanced minimum-norm estimation procedure has been proposed [38]. There is a need for research comparing the statistical properties of these definitions of the representation area. This can help develop guidelines for selecting an appropriate definition, possibly depending on the particular TMS mapping application.

The purpose of the present study was to determine the influence of the TMS mapping and data processing algorithms on the accuracy of estimating muscle representation parameters. Using a grid-based mapping approach, we studied the effect of MEP sampling, i.e. the size of the stimulation grid and the number of stimuli per cell, on the within-session accuracy and between-session variation of the muscle representation characteristics. We tested whether the between-session parameter changes could be explained by the withinsession MEP variability. Additionally, we investigated the impact of the data analysis methods by comparing several alternative definitions of the representation area, weighted area and center of gravity (COG) in terms of their estimation accuracy. The results can be applied for choosing an appropriate TMS mapping algorithm for a given research or clinical problem by finding a compromise between the accuracy requirements and mapping time constraints.

\section{Materials and Methods}

\subsection{Subjects and the nTMS mapping procedure}

For all subjects, an MRI was acquired in the T1 multiplanar reconstruction regime on a 3T Siemens MAGNETOM Verio clinical scanner. This data was used for TMS navigation.

The navigated TMS mapping was performed using the NBS eXimia Nexstim stimulator (Finland). We used a figure-of-eight biphasic coil with a diameter of $50 \mathrm{~mm}$ to deliver stimuli with a $280 \mu$ s duration. The maximum value of the estimated induced electric field in the cortex was $199 \mathrm{~V} / \mathrm{m}$. The electromyographic (EMG) activity of the studied muscles was recorded using skin pregelled disposable electrodes (Neurosoft, Russia). A suprathreshold MEP was defined as an EMG response having a peak-to-peak amplitude greater than or equal to $50 \mu \mathrm{V}$ in the interval from 15 to $30 \mathrm{~ms}$ after the stimulus. The individual resting motor threshold (RMT) was defined as the minimum intensity of stimulation for which five out of ten stimuli produced suprathreshold MEPs. The stimulation intensity during the mapping was set to $110 \%$ of the RMT.

Data from two experiments were employed for answering different research questions. The first dataset was recorded previously for different purposes. It was used here to determine an optimal size of the stimulation 
point grid for the second (main) experiment. The dataset contained 121 TMS maps for the abductor pollicis brevis (APB), extensor digitorum communis (EDC) and flexor digitorum superficialis (FDS) muscles of 33 healthy subjects ( 21 women, median age 27 , age quartiles 25,31 ; nine subjects were left-handed according to the Edinburgh handedness inventory [39]). In this experiment, the locations and sequence of the stimulation points were determined individually (without a grid), taking into account the responses obtained at previous points. Each point was stimulated once, and the mapping progressed in a given direction until obtaining two points without suprathreshold MEPs.

In the second experiment, the cortical representations of the right APB muscle in 8 healthy volunteers (3 women, median age 28, age quartiles 24, 29, all right-handed according to the Edinburgh handedness inventory [39]) were mapped three times on consecutive days. We used a stimulation point grid consisting of $7 \times 7$ square cells with a side of $7.63 \mathrm{~mm}$ (at the peeling depth of $20 \mathrm{~mm}$ ), centered at the hotspot. The cells were defined with the help of the grid tool in the Nexstim stimulator software. Ten rounds of stimulation were performed, and in each round, a single stimulus was applied to the center of every grid cell in a pseudorandom order ${ }^{1}$. The total number of stimuli in every session was 490 . All three sessions were performed with the same intensity equal to $110 \%$ of the individual RMT determined in the first session. The coil orientation was tangential to the surface of skull, and the induced electrical field was perpendicular to central sulcus, in the posterior to anterior direction.

The study was approved by the Ethical Committee of Research Center of Neurology (protocol 9-4/17, 30.08.2017), and written informed consent was obtained from all the participants.

\subsection{Data analysis}

\subsubsection{Muscle representation coverage by grids of different sizes}

Because the first dataset was acquired without a stimulation grid, the sizes of the obtained representations were not constrained from above and provided a sample from the size distribution in the healthy population. Thus, the maps were used to estimate the fractions of the representations that would be covered by square grids of different sizes centered at the point with the maximum MEP amplitude. Conservative estimates were used, counting only the parts of the representations that were guaranteed to be covered under any grid orientation (i.e. lying within a circle of a radius equal to half the side of the square). The calculations were performed for the following grid sizes: 38, 46, 53, 61 and $69 \mathrm{~mm}$ (corresponding to 10, 12, 14, 16 and 18 cells in the Nexstim grid tool) at the peeling depth of $20 \mathrm{~mm}$. The results were compared between the three muscles using the KruskalWallis test.

\subsubsection{Muscle representation parameters} A):

We calculated the following muscle representation parameters (the formulas are presented in Appendix

1. the area of the cells with the mean MEP above $50 \mu \mathrm{V}$;

2. the area of the cells with the maximum MEP above $50 \mu \mathrm{V}$ (or, equivalently, the area of the cells with at least one suprathreshold MEP);

3. the area of the cells with more than half suprathreshold MEPs;

4. the area weighted by the mean MEP amplitude (amplitude-weighted area, also known as map volume [17]);

5. the area weighted by the probability of a suprathreshold MEP (probability-weighted area);

6. the COG with the weights defined as the mean amplitudes in each grid cell;

7. the COG with the weights defined as the maximal amplitudes in each grid cell;

8. the COG with the weights defined as the probabilities of suprathreshold MEPs in each grid cell.

\subsubsection{Simulation of mapping with different numbers of stimuli using bootstrapping}

\footnotetext{
${ }^{1}$ In a small number of cases, due to operator error, the number of stimuli in a particular grid cell differed from ten, being equal to 9 in $7 \%$ of the cells, 11 in $4 \%$ and 7, 8 or 12 in less than $1 \%$ of the cells. The bootstrappingbased accuracy estimates did not significantly depend on such small variations, which was checked by repeating the calculations using the first eight stimuli in each cell for all the maps.
} 
To simulate the mapping results that would be obtained with a different number of stimuli per grid cell, we used a bootstrapping-based method, in which we randomly chose (with replacement) a given number of values from the 10 amplitudes measured in each cell. The resulting sets of amplitudes were treated as maps, and their parameters were calculated in the same way as for the initial full datasets. Sampling with replacement allows one to simulate arbitrary numbers of stimuli per cell (not necessarily smaller than 10). We performed the calculations for the numbers of stimuli from 1 to 10 . The last value corresponds to estimating the accuracy of the representation parameters for our actual protocol. The number of bootstrapping-generated maps was equal to 1000 for every condition.

\subsubsection{Bias of the area and weighted area}

An important and often overlooked fact is that the accuracy of an estimator is determined not only by its variance but also by the bias, i.e., the difference between the mean value of the estimator and the true value of the estimated parameter. It is necessary to characterize the bias because it can produce spurious effects and make the results obtained using different mapping protocols difficult to compare [40]. The evaluation of the bias is complicated by the inaccessibility of the 'true values' of the muscle representation parameters, i.e. those that would be obtained from a hypothetical mapping providing the full knowledge of the MEP probability distributions at every cortical location.

Our approach to estimating both the bias and variability of representation parameter estimates is based on bootstrapping [41]. Mathematically, the method simulates the mapping results for a muscle representation in which the actual probability distributions of MEP amplitudes in each cell coincide with the empirical distributions obtained in the experiment. It is important, however, that the validity of the estimates does not require exact equality between the empirical and the real MEP distributions, but is based on their approximate similarity, which can be expected with the ten-stimulus sampling.

The normalized (relative) bias was estimated by the following formula:

$$
B_{\text {norm }}(P)=\frac{\operatorname{mean}(P)-P_{0}}{P_{0}},
$$

where $P$ is a muscle representation parameter (such as the area), $P_{0}$ is the parameter value for the experimental map, and mean $(P)$ is the mean parameter value over the maps generated by bootstrapping with a certain number of stimuli per grid cell (ranging from one to ten).

\subsubsection{Within-session variability of the area and weighted area}

The within-session variability of muscle representation parameters was characterized by the coefficient of variation $(\mathrm{CV})$ of the parameter values for the maps generated by bootstrapping from a given experimental map:

$$
C V(P)=\frac{\operatorname{std}(P)}{\operatorname{mean}(P)},
$$

where $\operatorname{std}(P)$ is the sample standard deviation for the bootstrapping-generated maps.

\subsubsection{Between-session variability of the area and weighted area}

The variability of muscle representation parameters between the three mapping sessions was characterized by a variability index equal to one-half of the relative difference of the maximum and minimum values:

$$
V(P)=\frac{P_{\max }-P_{\min }}{P_{\max }+P_{\min }},
$$

where $P_{\max }$ and $P_{\min }$ are maximal and minimal values of the parameter in the three sessions. This quantity measures the relative deviation of these values from their mean. The values of this index were calculated and averaged by 1000 triples of maps generated by bootstrapping from the three mapping sessions.

\subsubsection{Sensitivity of the protocol to changes between sessions}

The MEP amplitudes in the three mapping sessions were compared in a cell-by-cell manner. Importantly, only the amplitudes above $50 \mu \mathrm{V}$ could be reliably detected. Thus, the values of all smaller responses were unknown - a situation called 'data censoring' in statistics [42]. Accordingly, the samples of MEP amplitudes from every grid cell were compared between the sessions using Gehan's generalization of the Mann-Whitney 
test for censored data [43]. To compare the within-session and between-session variability of MEPs, we performed similar tests between the two halves of each sample obtained in a given session (five MEPs in each half for every grid cell). To keep the same statistical power in the between-session tests, we limited them to the first half of each session (five MEPs per grid cell). The test results were visualized using 2D diagrams showing the locations of significant amplitude changes at uncorrected $\mathrm{p}<0.05$. The diagrams are analogous to statistical parametric maps in neuroimaging [44].

To assess whether the representation parameter changes between sessions had the same magnitude as the within-session fluctuations, the parameter distributions for maps generated by bootstrapping from each session were computed. The degree of similarity between pairs of distributions was measured by the overlaps of their histograms, with unit overlap corresponding to identical distributions and zero overlap - to completely incompatible distributions, with no common possible values. If the distributions in two sessions had a small overlap, this was interpreted as a significant change of the parameter between sessions, which could not be explained by the within-session variability.

In addition to the overlap values, it is useful to characterize the parameter heterogeneity in different sessions by a single number. To this end, we calculated the intraclass correlation coefficient (ICC) applied to the three parameter samples generated by bootstrapping from each session. We used the version of the ICC for the one-way random effects model [45] because the ordering of bootstrapping-generated maps is irrelevant. We call the resulting quantity the bootstrapping-based between-session intraclass correlation coefficient (BICC). In a given subject, this index measures the proportion of the parameter variance attributable to systematic session differences. Zero BICC corresponds to a situation in which the changes between sessions can be fully explained by the variability within a session, and high BICC indicates stronger variation between than within sessions. This measure should be distinguished from the ICC applied in the way common in reliability studies, where it is computed for the sets of values obtained in different subjects and quantifies the ability to distinguish the characteristics of different individuals in the presence of variability [45]. Conversely, BICC is calculated for a single subject and measures the ability to discriminate between sessions in the presence of within-session inaccuracy.

\subsubsection{Accuracy of the center of gravity}

The accuracy of the COG was measured by the mean distance between the COG calculated from the experimental map and the COGs of 1000 maps generated by bootstrapping.

\section{Results}

\subsection{Muscle representation coverage by grids of different sizes}

For every percentage value $\mathrm{X}$, we calculated the fraction of all healthy subjects for whom at least $\mathrm{X}$ per cent of their representation is covered by the grid of a given size (Fig. 1). This analysis was performed for the maps from the first dataset obtained without a grid. The coverage fractions were not significantly different between the three muscles (APB, EDC and FDS) for every grid size ( $p>0.05$, Kruskal-Wallis test). Based on this analysis, we selected for the main experiment a grid size of $53 \mathrm{~mm}$ (14 cells in the Nexstim grid tool), covering on average $97.9 \%$ of the area of the representations. 


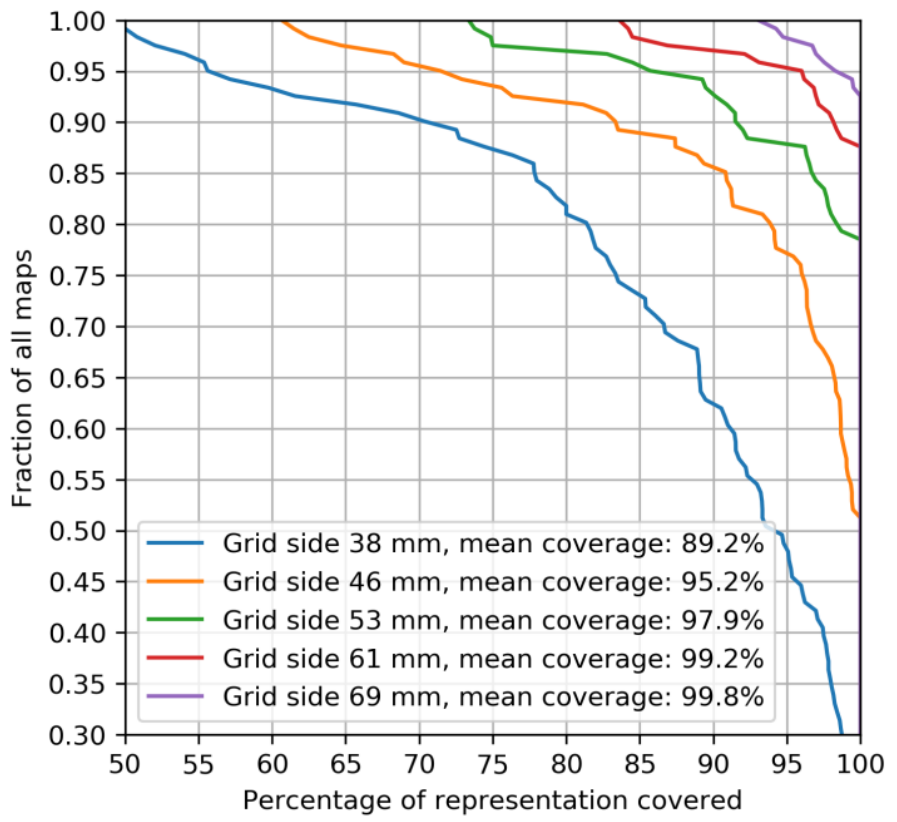

Figure 1. The effect of the grid size on the completeness of muscle representation coverage. For every percentage value $\mathrm{X}$, the corresponding $\mathrm{Y}$ value is the fraction of all the maps in which at least $\mathrm{X}$ per cent of the representation is covered by the grid of a given size. The simulated grids were located at the peeling depth of $20 \mathrm{~mm}$, centered at the point with the maximum MEP amplitude, had a square shape, and their side lengths were chosen as even integer multiples of a cell in the Nexstim grid tool (10 to 18 cells). The TMS maps used in this calculation were obtained without a grid in healthy subjects (13 maps for APB, 54 for EDC, and 54 for FDS). There was no significant difference in the representation size between the muscles for every grid size ( $>0.05$, Kruskal-Wallis test). Note: The estimates of the coverage are conservative in that stimulation points were counted as covered by the grid only if their distance from the hotspot was smaller than one-half of the side of the grid (i.e. excluding the coverage by the corners of the square, which is possible, but not guaranteed under varying grid orientations). The green line corresponds to the grid size used in the present study.

\subsection{Visualization of TMS maps obtained with a stimulation grid}

The mapping results from the grid-based experiment were visualized by representing each grid cell by a The muscle representations were generally composed of a region of varying size having a high probability of a suprathreshold response ( 0.9 and above, colored yellow) and a surrounding area with an intermediate probability (ranging from 0 to 0.9 , colored green to dark violet). 


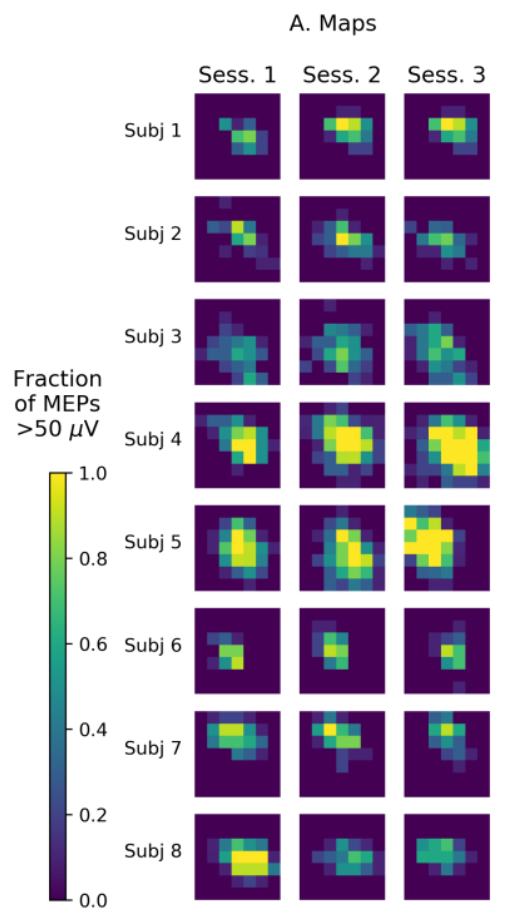

B. Differences between sessions
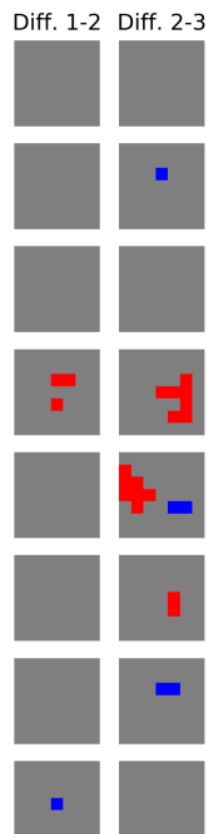
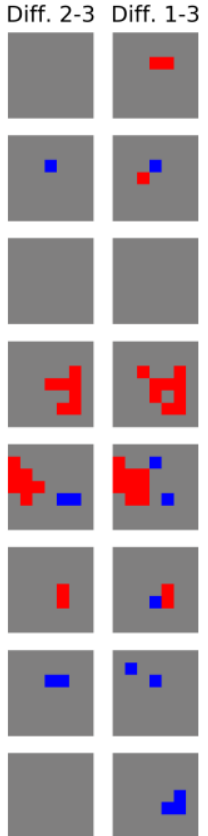
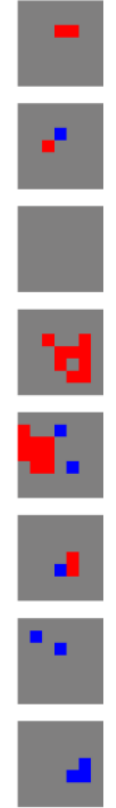

C. Differences within sessions (between halves of a session)

Diff. 1 Diff. 2 Diff. 3
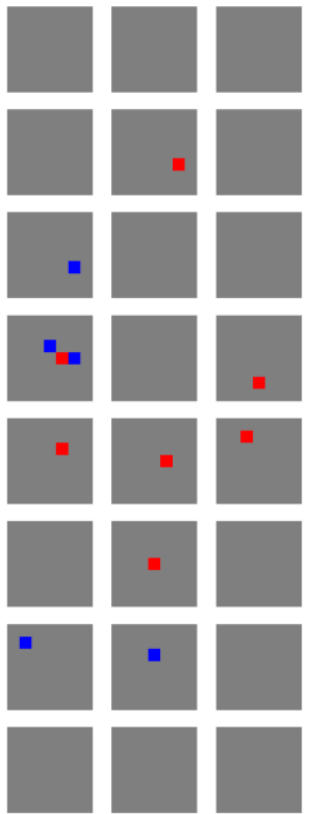

Figure 2. A. TMS maps of the APB muscle from three sessions performed on consecutive days. The squares represent the stimulation grid cells, and the color encodes the fraction of the applied 10 stimuli that produced suprathreshold MEPs (above $50 \mu \mathrm{V}$ ). B. Results of the comparison of the first five MEP amplitudes in each cell between sessions using Gehan's generalization of the Mann-Whitney test for censored data. The red cells had significantly greater amplitudes in the second session of the compared pair (with uncorrected $\mathrm{p}<0.05$ ), and the blue cells - significantly smaller amplitudes. C. Results of the comparison of the first and the second five MEP amplitudes in each cell. The test and the color code are the same as in B.

\subsection{Bias of the area and weighted area}

The biases of the different variants of the area and weighted area were calculated using the bootstrappingbased map simulation, and their median values from all sessions in all subjects are shown in Fig. 3 as functions of the number of stimuli per grid cell. A considerable bias exists in the (unweighted) area parameters, which were defined using thresholding: the area of the cells with the mean MEP above $50 \mu \mathrm{V}$, the area of the cells with the maximum MEP above $50 \mu \mathrm{V}$ and the area of the cells with more than half suprathreshold MEPs. In contrast, the amplitude-weighted area and probability-weighted area have very small biases.

For the area of the cells with more than half suprathreshold MEPs, the bias showed different patterns for even and odd numbers of stimuli per cell, shown separately by the solid and dashed green lines respectively. Moreover, as shown in Appendix C, for particular structures of the representations, the bias of this parameter can be a non-monotonic function of the number of stimuli. The sign of the bias can be negative or positive, depending in a non-trivial way on the details of the representation and the number of stimuli. This suggests interpreting this parameter with caution. 


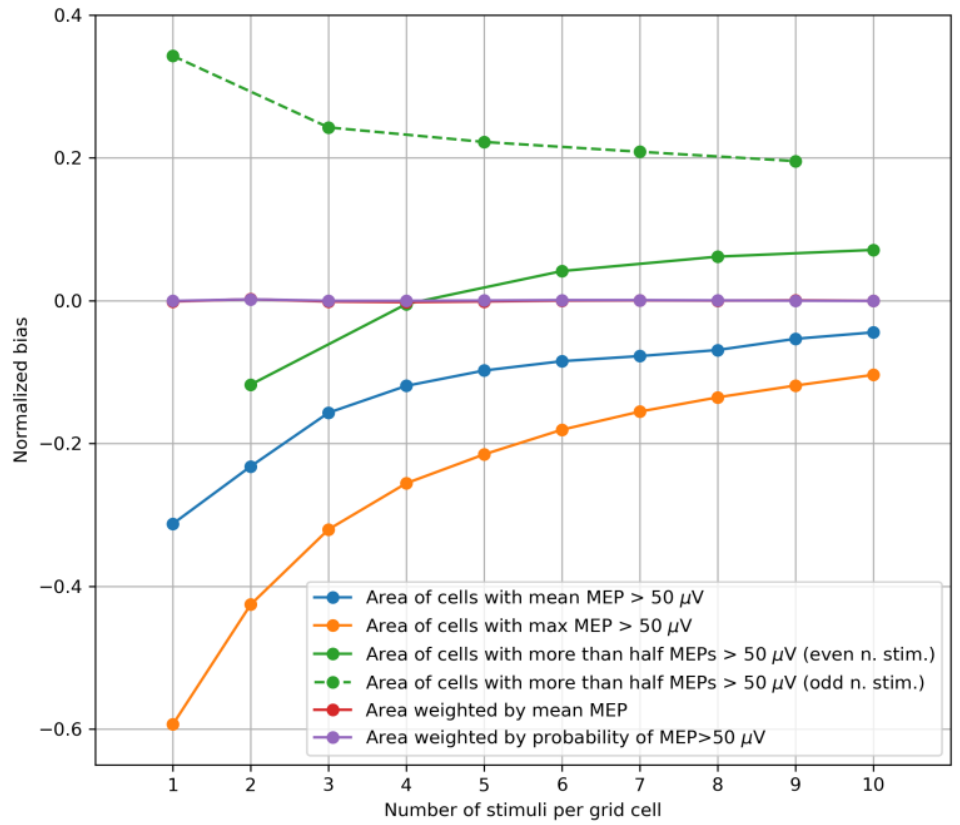

Figure 3. The dependence of the normalized bias of the representation parameters on the number of TMS stimuli per grid cell. The values of the parameters were averaged by 1000 maps generated by bootstrapping (with replacement) from every experimental map obtained with 10 stimuli per cell in each subject. The median values from all maps of all subjects are depicted. The area of the cells with more than half suprathreshold MEPs showed different patterns for even and odd numbers of stimuli per cell, shown by the solid and dashed green lines respectively (here and in Figs. 4, 5). The biases of the mean amplitude-weighted and probability-weighted areas (red and purple curves) are close to zero.

\subsection{Within-session variability of the area and weighted area}

The within-session CVs were calculated using the same method as the biases and plotted depending on number of stimuli per grid cell in the maps generated by bootstrapping (Fig. 4). For all the parameters, the CV significantly decreased with the number of stimuli per cell ( $\mathrm{p}<0.001$, Page's trend test for ordered alternatives). The parameters having the smallest CVs were the area of the cells with at least one suprathreshold MEP and the probability-weighted area.

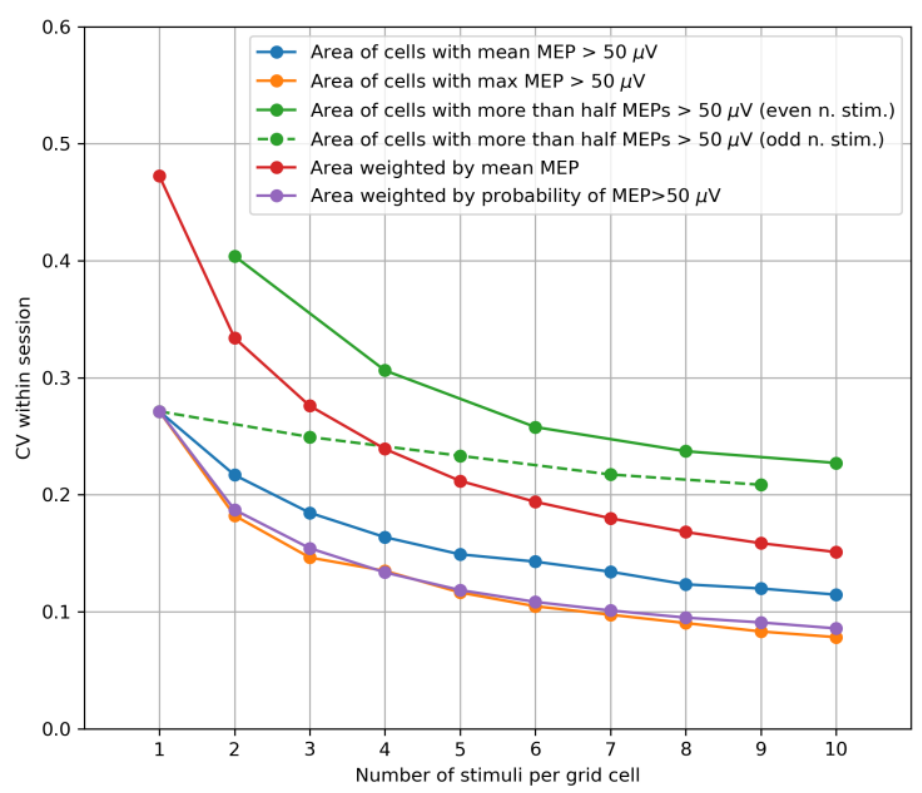


Figure 4. Within-session variability of area parameters measured by the coefficient of variation (CV) of the parameter values obtained in 1000 maps generated by bootstrapping from every initial 10-stimuli-per-cell map of every subject. The median values of the CVs from all maps of all subjects are depicted. For all the parameters, the CV significantly decreases with the number of stimuli per cell $(\mathrm{p}<0.001$, Page's trend test for ordered alternatives).

The probability-weighted area was characterized by the highest overall accuracy among the considered definitions of the area and weighted area, having a negligible bias and a small CV. This parameter was selected for further analysis of its sensitivity to the between-session map changes (section 3.6).

\subsection{Between-session variability of the area and weighted area}

The between-session variability index demonstrated a pattern similar to that of the within-session CV (Fig. 5 ). The variability index significantly decreases with the number of stimuli per cell ( $\mathrm{p}<0.001$, Page's trend test) for all the parameters except the area of the cells with more than half suprathreshold MEPs, which can have a non-monotonic, subject-dependent bias and should be interpreted with caution (see Appendix C). The parameters with the smallest between-session variability were the area of the cells with at least one suprathreshold MEP and the probability-weighted area (the same parameters that had the smallest withinsession $\mathrm{CV}$ ) as well as the area of the cells with the mean MEP above $50 \mu \mathrm{V}$.

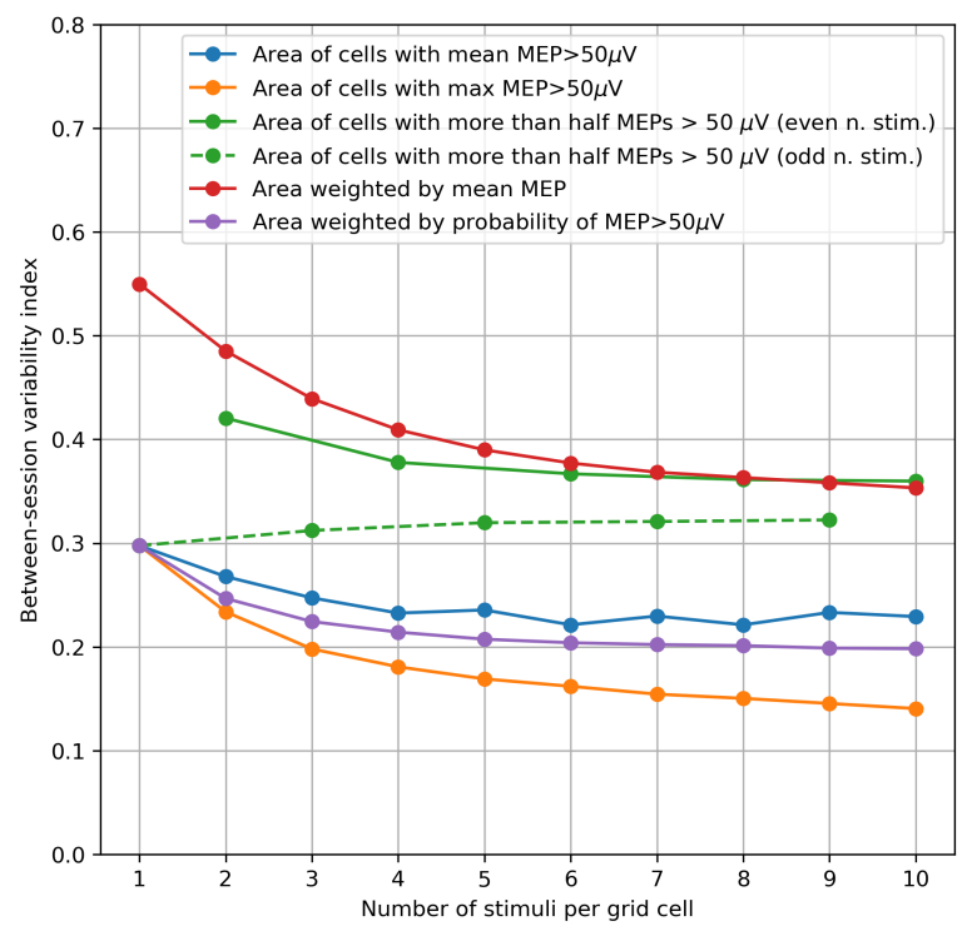

Figure 5. Between-session variability of the area parameters measured by an index equal to one-half of the relative difference of the maximum and minimum values among the three mapping sessions performed on consecutive days. These indices were calculated and averaged by 1000 triples of maps generated by bootstrapping from the MEPs obtained in the three sessions. The median values from all subjects are shown in the plot. The variability index significantly decreases with the number of stimuli per cell $(p<0.001$, Page's trend test) for all the parameters except the area of the cells with more than half suprathreshold MEPs, which can have a non-monotonic, subject-dependent bias and should be interpreted with caution (see Appendix C).

\subsection{Sensitivity of the protocol to changes between sessions}

The results of the amplitude comparisons for each grid cell between the first halves of pairs of sessions and between the first and second halves of each session indicate that, on average, the number of significant changes was greater between sessions than within a session (Fig. 2 B,C). 
The relationship between the within-session and between-session variability of the probability-weighted area was characterized by calculating its probability distributions for the maps generated by boostrapping from each session (Fig. 6). Five of the eight subjects had a between-session distribution overlap of less than 0.05 in at least one pair of sessions, indicating a significant difference in the probability-weighted area.

We quantified the ability to distinguish the values of the probability-weighted area between sessions using the BICC, i.e. the intraclass correlation coefficient applied to the three parameter samples generated by bootstrapping from each session. The BICC ranged from 0.61 to 0.99. High BICC values (above 0.9) were observed in the three subjects (with numbers 4, 5 and 8) who had zero distribution overlaps in some pairs of sessions. Both measures indicate that in these subjects, the between-session changes of the probability-weighted area were greater than the within-session fluctuations and thus were unlikely to be explainable solely by the trial-to-trial variability of MEPs.
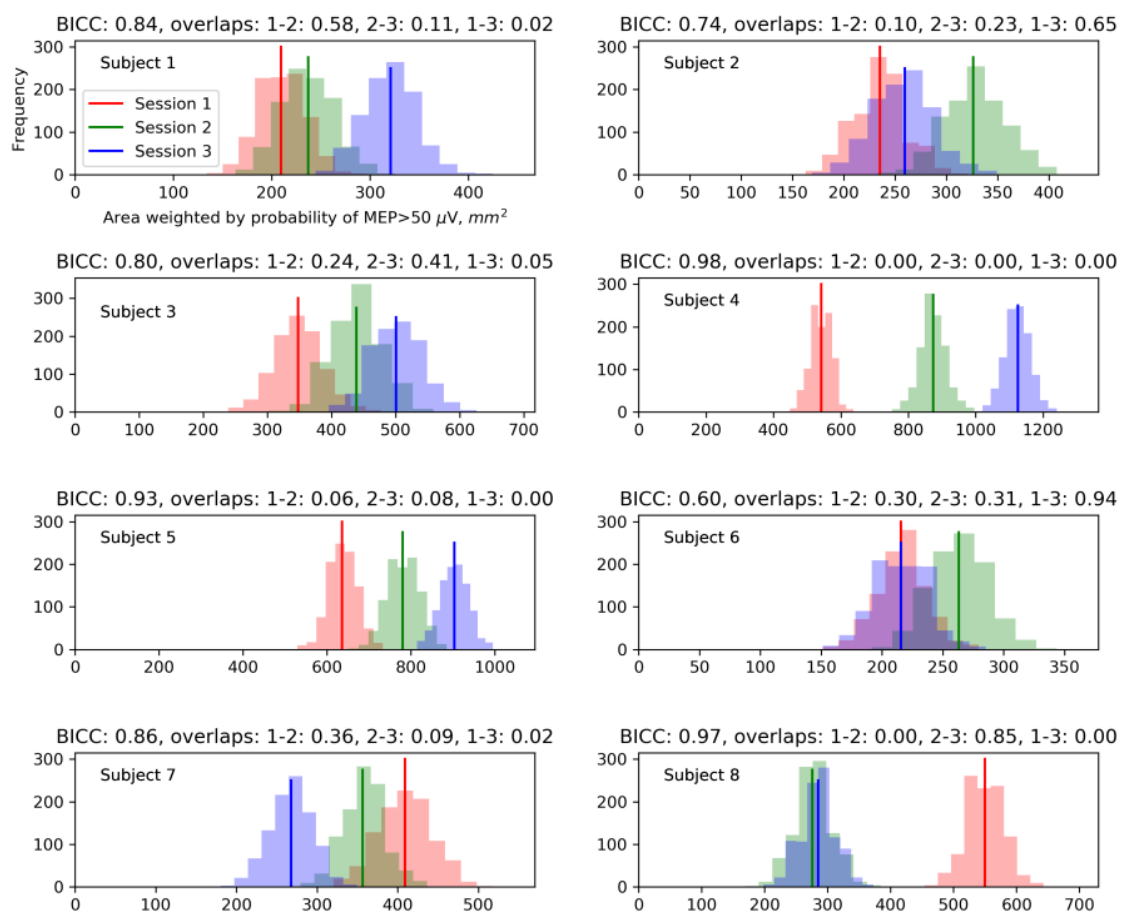

Figure 6. Comparison of the between-session and within-session variability for the probability-weighted area (sum of grid cell areas multiplied by the probabilities of suprathreshold MEPs in them). Each plot corresponds to one subject and shows three histograms for different mapping sessions. Each histogram shows the withinsession distribution of the values of the probability-weighted area obtained from 1000 maps generated by bootstrapping from a given map. Above the plots, the measures of the possibility to discriminate between sessions are shown: the bootstrapping-based between-session intraclass correlation coefficient (BICC) and the pairwise distribution overlaps.

Additionally, to compare the alternative definitions of the area and weighted area by their ability to find significant differences between sessions at the individual level, we computed the BICC values for all the parameters and all subjects (Fig. 7). In five of the eight subjects, the highest BICC was shown by the probabilityweighted area, and in the remaining three subjects - by the amplitude-weighted area. 


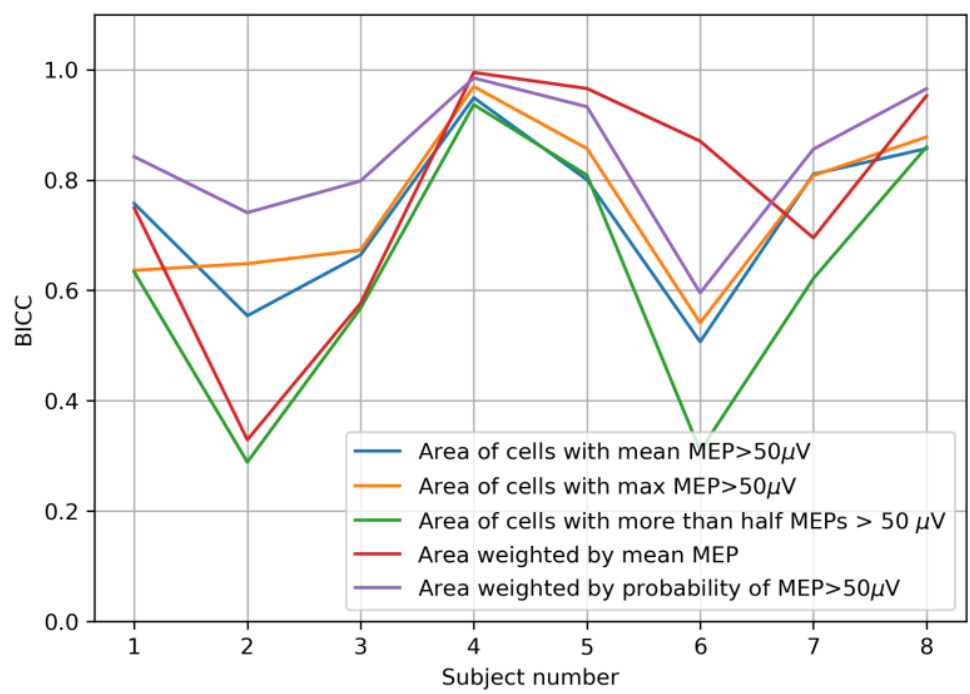

Figure 7. Bootstrapping-based between-session intraclass correlation coefficient (BICC) calculated for all the area and weighted area variants in all subjects.

\subsection{Accuracy of the center of gravity}

The COG accuracy was measured by the mean distance between the COG calculated from the initial map with 10 stimuli per cell and the COGs of 1000 maps generated by bootstrapping. The results are shown in Fig. 8 depending on number of stimuli per grid cell in the bootstrapping-generated maps. For all the COG variants, this error measure significantly decreased with the number of stimuli ( $<<0.001$, Page's trend test). The highest accuracy was obtained for the probability-weighted COG, although the accuracy differences with the other two definitions were small (less than $1 \mathrm{~mm}$ ).

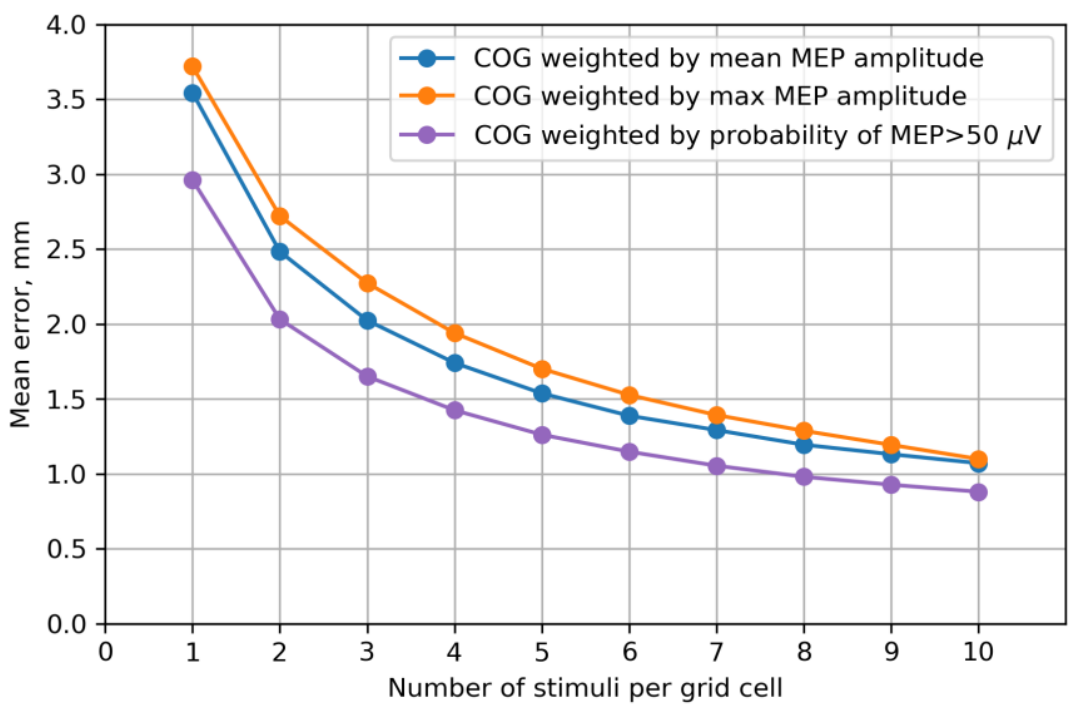

Figure 8. Accuracy of the COG estimates computed using three alternative methods of assigning weights to the stimulation points. The accuracy is measured by the mean distance between the COG calculated from the full map and the COGs of 1000 maps generated by bootstrapping. The median values from all maps of all subjects are shown. The differences in the COG accuracy between the three methods are statistically significant for all numbers of stimuli smaller than 8 ( $p<0.05$, Friedman test). The highest accuracy is achieved by the approach in which the stimulus location vectors are weighted by the probability of a suprathreshold MEP in them (purple curve), although the accuracy differences between the methods are small (less than $1 \mathrm{~mm}$ ). For all the COG variants, the error significantly decreases with the number of stimuli per cell ( $p<0.001$, Page's trend test). 


\section{Discussion}

We have studied the impact of the TMS mapping algorithm and data processing on the accuracy of estimating muscle representation parameters. The considered aspects of the mapping procedure were the size of the stimulation grid and the number of stimuli per cell. As regards data processing, several alternative definitions of the muscle representation area, weighted area and COG were compared in terms of the accuracy of their estimation. Among the considered variants of area and weighted area, the highest overall accuracy was shown by the area weighted by the probability of a suprathreshold MEP. This parameter was further investigated with respect to its sensitivity to the motor map changes between the three sessions recorded on consecutive days. The results show that such changes can be greater than the fluctuations within a session, and thus can be reliably detected in individual subjects using the present protocol. The causes of these changes, including possible physiological and methodological explanations, require further research.

\subsection{Muscle representation coverage by grids of different sizes}

The optimal choice of the stimulation grid size has rarely been discussed in the literature. Classen et al. [20] calculated the increasing accuracy of the COGs obtained using square grids with side lengths of 3,5 and 7 $\mathrm{cm}$. Since the main expected effect of an insufficiently large grid is likely to be the missing of some excitable sites at the periphery, we based our analysis on the percentage of the points with suprathreshold MEPs covered by a grid. The obtained dependence of this characteristic on the grid dimensions can be used to choose an appropriate size that is large enough to ensure the required representation coverage. At the same time, an unnecessarily large grid is undesirable due to the increased mapping time (if the stimulation point density is fixed).

\subsection{Visualization of TMS maps obtained with a stimulation grid}

The mapping protocol used in this study produced samples of 10 MEP amplitudes from every grid cell in each session. This allowed a statistical comparison of the maps in a cell-by-cell manner - an approach that is widespread in MRI-based neuroimaging, but not so common in TMS mapping (although is occasionally applied [7]). We found a considerable number of significant changes of amplitude distributions between sessions and visualized the spatial configurations of these effects. Significant changes between sessions were more numerous than alterations within a session (i.e. between its first and second halves). This motivates further application of the described methodology for testing location-specific MEP changes with and without interventions based on the MEP samples of considerable size obtained in each grid cell in different mapping sessions.

\subsection{Bias of the area and weighted area}

One of the problems in the field of TMS mapping is the difficulty of comparing results obtained by different groups using a variety of mapping protocols and data processing methods. The performed analysis of the biases of the different variants of area and weighted area indicates that the values of the thresholding-based (unweighted) area definitions have considerable biases. This means that these parameters can systematically differ between protocols with different numbers of stimuli per grid cell. Additionally, every subject is characterized by a particular bias, depending on the details of the MEP probability distributions in all the grid cells (see Appendix B). This means that the influence of the bias cannot be eliminated by a single bias correction procedure. Moreover, if a study applying TMS mapping with a limited number of stimuli compares the representation areas in two groups with systematically different area biases, a totally spurious difference in the area can be obtained. The amplitude-weighted and probability-weighted areas have negligible biases, and thus do not present the above problems.

It should be stressed, however, that the choice of the parameters to focus on in a given study cannot be based solely on their accuracy. Indeed, a parameter may be estimated very accurately, but show no effect in the considered problem. Thus, all muscle representation characteristics can potentially be informative, particularly if their statistical properties are understood and taken into account.

\subsection{Within-session variability of the area and weighted area}

The extreme variability of MEP amplitudes (which can span more than two orders of magnitude [46]) leads to the within-session variability of muscle representation parameters [35]. The characteristics considered 
here involve integration of data from repeated stimulation of many cortical locations, which makes the representation parameters more stable than a single MEP. This effect of stabilization due to averaging was found to vary depending on the exact definition of the representation area or COG. We estimated the variability using a bootstrapping-based method, in which we simulated maps by subsampling MEPs from the datasets recorded in the experiment.

The results show that the three alternative definitions of the muscle representation area produce different degrees of relative variability (measured by the CV). One of the variants is the area of the cells with at least 6 suprathreshold MEPs (out of 10 stimuli), which was recommended in the protocol proposed in [21] and named "the golden standard" in [40]. This parameter had a larger CV than the other two (unweighted) area variants: the area of the cells with the mean MEP above $50 \mu \mathrm{V}$ and the area of the cells with at least one suprathreshold MEP. As noted above, this does not imply that any of the parameters should not be used, because although all the three area variants depend on the representation extent, they do not measure exactly the same property and may be sensitive to different effects of interest. The higher variability of the area of the cells with at least 6 suprathreshold MEPs leads to the requirement of larger effect sizes and/or samples for statistical significance as compared to the other two area definitions. Thus, it is important to take into account the accuracy of the different parameters for planning the experiments, even though the accuracy cannot serve as the only basis for parameter selection.

The probability-weighted area has the highest overall accuracy among the area and weighted area variants. It depends on both the extent of the representation and the distributions of MEPs at the included points. Further studies are warranted to assess the utility of this parameter in fundamental and clinical problems.

The obtained decreasing dependencies of errors on the number of stimuli per grid cell can be used for appropriately choosing this number in a given application of TMS mapping. A compromise should be reached between the requirements for high accuracy and reasonable study duration. Several methodological studies of TMS mapping have focused on the number of stimuli sufficient for reliable estimation of representation parameters [20,26,34,36,40,47], and their results may be considered to mean that any further increase in this number is pointless. The obtained dependencies (Fig. 4) demonstrate that, although the slopes are largest in the left parts of the curves, the errors continue to decrease for all the considered numbers of stimuli. Thus, a study with a small effect size may benefit from a larger number of stimuli than the minimum one required for reliability.

High parameter accuracy may be especially relevant to investigations of the changes in TMS maps between two time points due to an intervention or spontaneous directed alteration such as disease progression. In such a study, the measured change of a parameter is composed of (1) the constant mean effect of interest, (2) the random change in the mean parameter value between the sessions and (3) the within-session random errors. The error terms (2) and (3) can contain both physiological components (such as excitability fluctuations) and methodological factors (e.g., navigation inaccuracies). The ability to detect the effect (i.e. the statistical power) depends on its size in relation to the error terms (2) and (3). The purpose of sufficient MEP sampling studied in this paper is to reduce the component (3) so that it is small compared to the component (2) and thus does not limit the statistical power. Meanwhile, it is known that the between-session variability (2) is smaller than the between-subject variation, as indicated by the reported ICC values above $0.5[47,48]$. Thus, even a small accuracy gain irrelevant for group comparisons may be essential in pre-post studies.

\subsection{Between-session variability of the area and weighted area}

Similarly to the within-session $\mathrm{CV}$, the between-session variability indices of the area and weighted area decreased with the number of stimuli. However, this decrease showed a more pronounced flattening for numbers of stimuli greater than five, in comparison with the decrease of the CV. This is in agreement with the interpretation that increasing the number of stimuli reduces the effect of the short-term MEP variability and brings representation parameters closer to their mean values in a particular session, but these mean values may differ between sessions due to physiological and/or methodological factors. This means that the betweensession variability will approach a (nonzero) plateau determined by the differences between the mean values in the sessions. In other words, it is impossible to eliminate the between-session changes by collecting more data in each session.

The relationships between the alternative area definitions were similar to those observed for the withinsession CV, with two exceptions. First, the CV was smaller for the amplitude-weighted area than the area of the cells with more than half suprathreshold MEPs, whereas their between-session variability indices were 
similar. Second, the between-session variability was higher for the probability-weighted area than the area of the cells with at least one suprathreshold MEP, whereas their CVs were similar. This may correspond to a greater day-to-day stability of the representation 'footprint' (the area of the region able to produce MEPs) than its 'height' in terms of MEP probability (i.e. the average degree of certainty with which a suprathreshold response will be elicited in each location).

\subsection{Sensitivity of the protocol to changes between sessions}

An optimal TMS mapping protocol for a given study must be sensitive to the effect being investigated. As mentioned above, an important type of research question concerns the changes of TMS maps with time, e.g. in the course of disease progression [14] or as a result of neuroplasticity caused by therapeutic interventions [15]. To reliably detect such changes at the level of individual subjects, the within-session variability should be small compared to the between-session effect size. In the present study, we compared three mapping sessions without any interventions between them. The changes in the probability-weighted area between the consecutive days were shown to be greater than the within-session fluctuations. This suggests that the day-to-day changes in this parameter cannot be fully explained by the inaccuracy produced by the trial-to-trial MEP amplitude variability.

\subsection{Accuracy of the center of gravity}

Similarly to the $\mathrm{CV}$ of the extent-related representation parameters, the within-session errors in the center of gravity decreased with the number of stimuli. They were within the nominal accuracy of the navigation system $(5.7 \mathrm{~mm})$. The probability-weighted COG showed a slightly higher accuracy than the COGs weighted by the mean and maximum MEPs, which may be due to its independence of large fluctuations in the MEP amplitudes known to have a heavy-tailed distribution [46].

\section{Conclusions}

We have studied the dependence of the accuracy of muscle representation parameters on the aspects of the grid-based TMS mapping experiment and data processing. The grid size impacted the completeness of the muscle representation coverage, and a square grid with a side of $53 \mathrm{~mm}$ (at the peeling depth of $20 \mathrm{~mm}$ ) centered at the hotspot covered on average $97.9 \%$ of the representation area for the APB, EDC and FDS muscles. The within-session accuracy of the representation area, weighted area and COG improved with the increasing number of stimuli without saturation up to at least ten stimuli per cell. For the area definitions based on thresholding, a considerable bias was observed for small numbers of stimuli, while for the probability-weighted and mean amplitude-weighted areas the bias was negligible. The area weighted by the probability of a suprathreshold MEP showed the highest overall accuracy among the considered definitions of the area and weighted area (surpassing the accuracy of the commonly considered area of the cells with more than half suprathreshold MEPs). The protocol was found to have sufficient sensitivity to distinguish the between-session changes of the probability-weighted area from its within-session fluctuations. The results can guide the choice of the grid size, the number of stimuli per cell and the investigated representation parameters in studies applying TMS mapping to research and clinical problems.

Data and code availability: The TMS mapping data and the source code of the scripts used for data processing are available at https://github.com/DOSinitsyn/gridTMSmaps.

Funding: This research was funded by the Russian Science Foundation, grant number 17-75-10062.

Conflicts of Interest: The authors declare no conflict of interest.

\section{Appendix A. Formulas for the muscle representation parameters}

The muscle representation parameters were defined by the following formulas:

1. The area of the grid cells with the mean MEP amplitude above $50 \mu \mathrm{V}$ :

$$
A_{\text {mean thr }}=A_{\text {cell }} N_{\text {mean } t h r} \text {. }
$$


Here $A_{\text {cell }}$ is the area of a grid cell, and $N_{\text {mean thr }}$ is the number of cells for which mean $\left(M_{i}\right)>t$, where $M_{i}$ represents the MEP amplitudes obtained in a cell, and $t$ is the amplitude threshold (50 $\left.\mu \mathrm{V}\right)$. The number of the averaged amplitudes $M_{i}$ is the number of stimuli per cell $n_{\text {stim }}$, which was equal to ten in our experimental maps and ranged from one to ten in the bootstrapping-generated maps.

2. The area of the cells with the maximum MEP above $50 \mu \mathrm{V}$ (or, equivalently, the area of the cells with at least one suprathreshold MEP):

$$
A_{\text {max thr }}=A_{\text {cell }} N_{\text {max thr }},
$$

where $N_{\text {max }}$ thr is the number of cells for which $\max \left(M_{i}\right)>t$.

3. The area of the cells with more than half suprathreshold MEPs:

$$
A_{\text {half thr }}=A_{\text {cell }} N_{\text {half thr }},
$$

where $N_{\text {half } t h r}$ is the number of cells for which more than half of the stimuli produced amplitudes $M_{i}>t$.

4. The area weighted by the mean MEP amplitude (amplitude-weighted area):

$$
A_{\text {mean } w}=A_{\text {cell }} \sum_{\text {cells }} \operatorname{mean}\left(M_{i}\right) \text {. }
$$

5. The area weighted by the probability of a suprathreshold MEP (probability-weighted area);

$$
A_{\text {prob } w}=A_{\text {cell }} \sum_{\text {cells }} \mathrm{p}\left(M_{i}>t\right),
$$

where $\mathrm{p}\left(M_{i}>t\right)$ is the fraction of all the amplitudes in a cell that are greater than $t$.

6. The COG with the weights defined as the mean amplitudes in each grid cell:

$$
\vec{C}_{\text {mean } w}=\sum_{\text {cells }}\left(\operatorname{mean}\left(M_{i}\right) \vec{r}\right) / \sum_{\text {cells }} \operatorname{mean}\left(M_{i}\right),
$$

where $\vec{r}$ is the position vector of a cell, mean $\left(M_{i}\right)$ is the mean MEP amplitude in this cell.

7. The COG with the weights defined as the maximal amplitudes in each grid cell;

$$
\vec{C}_{\text {max } w}=\sum_{\text {cells }}\left(\max \left(M_{i}\right) \vec{r}\right) / \sum_{\text {cells }} \max \left(M_{i}\right) .
$$

8. The COG with the weights defined as the probabilities of suprathreshold MEPs in each grid cell.

$$
\vec{C}_{\text {prob } w}=\sum_{\text {cells }}\left(\mathrm{p}\left(M_{i}>t\right) \vec{r}\right) / \sum_{\text {cells }} \mathrm{p}\left(M_{i}>t\right) .
$$

\section{Appendix B. Biases of the area and weighted area for the individual maps}

The biases of the area and weighted area variants displayed considerable dependence on the details of the MEP distributions in a particular TMS map. Figs. 9-13 show the biases of the extent-related representation parameters for each of the three sessions in every subject. The heterogeneity of the bias of each parameter precludes its elimination by a universal bias correction procedure. 
bioRxiv preprint doi: https://doi.org/101101/573220; this version posted March 12, 2019. The copyright holder for this preprint (which was not certified by peer review) is the author/funder. All rights reserved. No reuse allowed without permission.

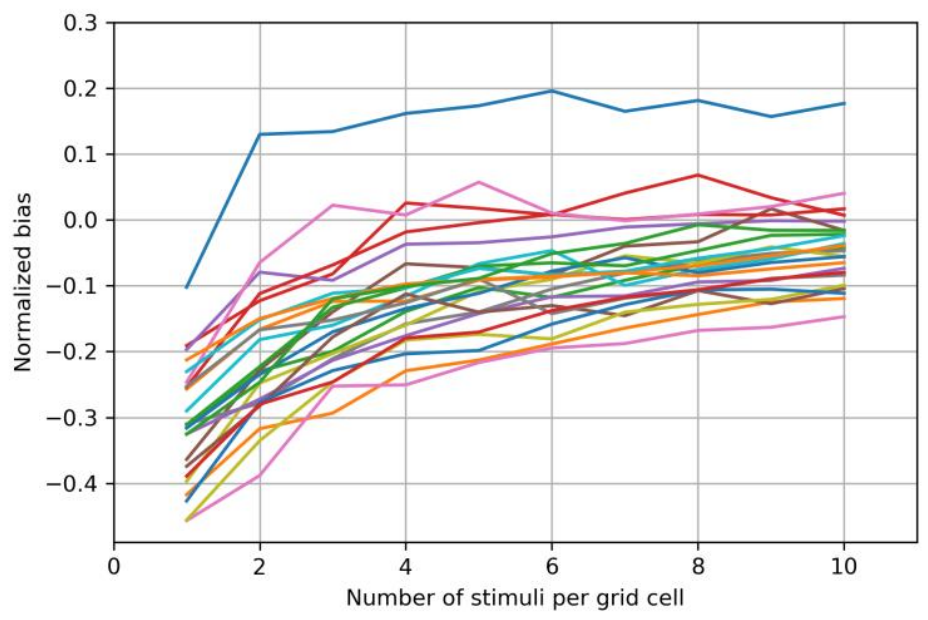

Figure 9. Bias of the area of the cells with the mean MEP above $50 \mu \mathrm{V}$ for each TMS map obtained in a given session of a particular subject, shown for all subjects and sessions. Here and below, every map corresponds to one line in the plot.

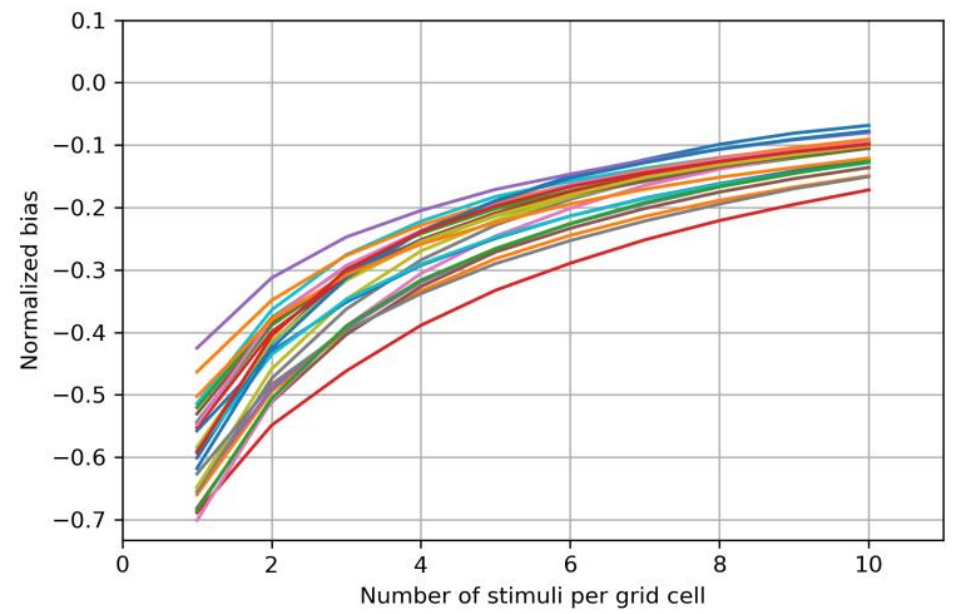

Figure 10. Bias of the area of the cells with the maximum MEP above $50 \mu \mathrm{V}$ for all sessions of all subjects.

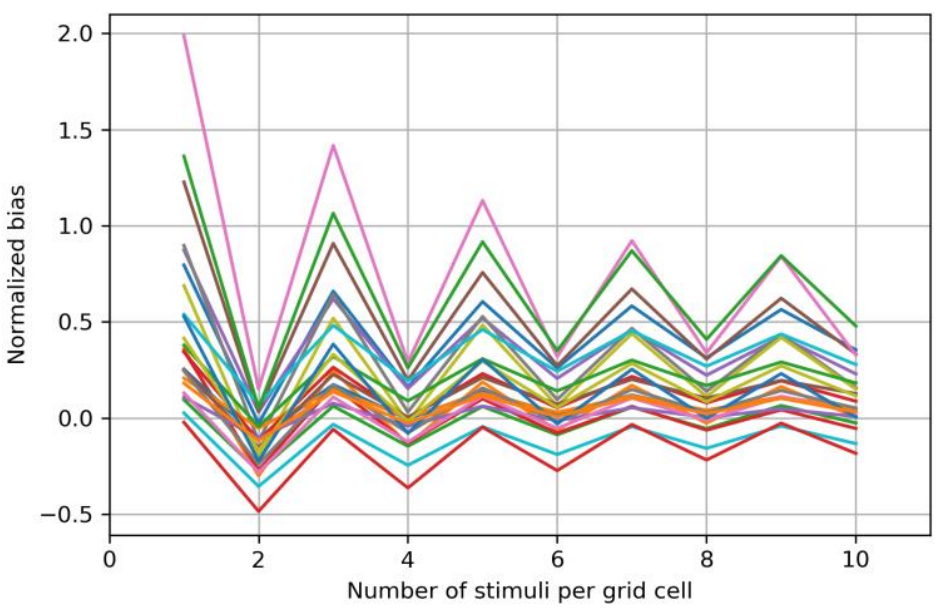

Figure 11. Bias of the area of the cells with more than half suprathreshold MEPs for all sessions of all subjects. 


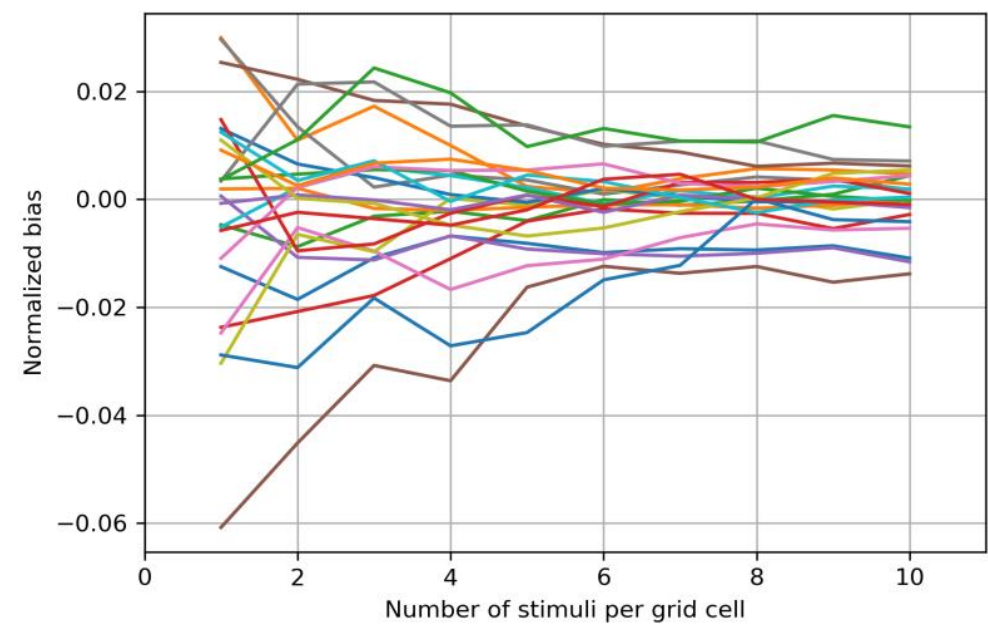

Figure 12. Bias of the area weighted by the mean MEP amplitude for all sessions of all subjects.

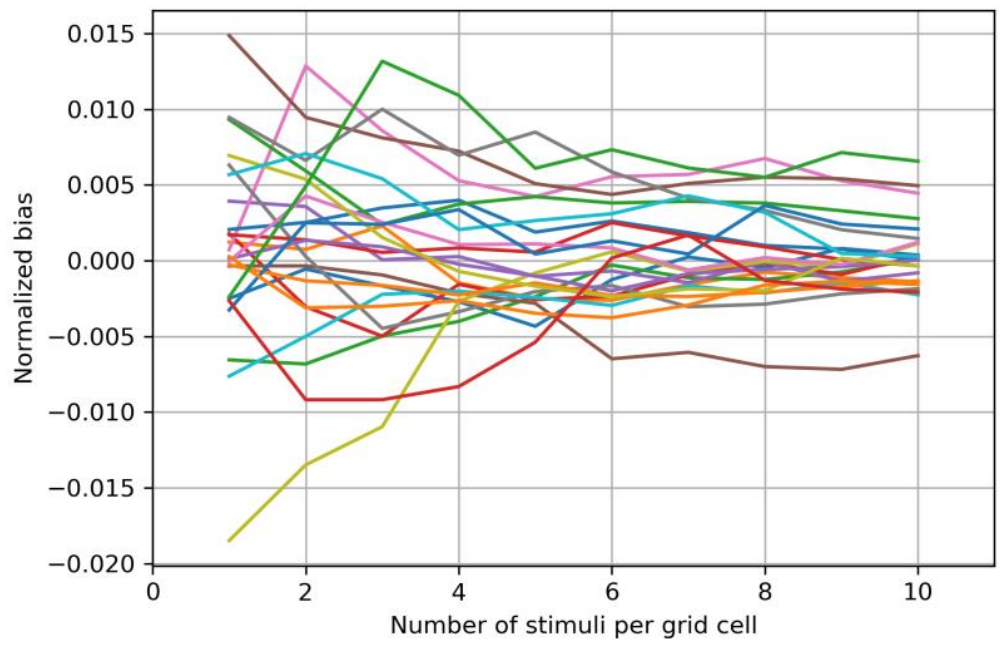

Figure 13. Bias of the area weighted by the probability of a suprathreshold MEP for all sessions of all subjects.

\section{Appendix C. Bias structure of the area of the cells with more than half suprathreshold MEPs}

Investigation of the area of the cells with more than half suprathreshold MEPs $\left(\mathrm{A}_{\text {half }}\right)$ showed that this parameter has unintuitive statistical properties for particular structures of muscle representations. We illustrate this with the following example. Consider a muscle representation consisting of two grid cells of unit area, with the probabilities of suprathreshold MEPs equal to 0.4 and 0.7 . Since one of the cells has the probability above 0.5 , the true value of $A_{\text {half }}$ is equal to 1 . The mapping of this representation with $n_{\text {stim }}$ stimuli per cell produces samples of the size $n_{\text {stim }}$ from the Bernoulli distributions with the success probabilities of 0.4 and 0.7 . The number of suprathreshold MEPs in each cell is the corresponding binomial variable. Let us define two random variables that are equal to 1 if the corresponding binomial variable is greater than $0.5 * \mathrm{n}_{\text {stim }}$ and 0 otherwise. These variables are Bernoullian, and their success probabilities can be calculated from the binomial distributions. The estimate of $A_{\text {half }}$ obtained from the mapping is the sum of these variables.

Fig. 14A shows the dependence of the bias of the estimate of $A_{\text {half }}$ on the number of stimuli per cell, $n_{\text {stim. }}$ The oscillations correspond to the difference in the bias between the even and odd numbers of stimuli. If we restrict this number to be even (or odd), the dependence remains non-monotonic, with an initial increase followed by a decrease. This pattern is explained by the fact that the area estimate is the sum of the contributions of each cell. The first cell (with the suprathreshold MEP probability of 0.4) produces a mean area estimate that slowly decreases with $\mathrm{n}_{\text {stim }}$ (Fig. 14B), whereas the contribution of the second cell (having probability 0.7) shows a faster increase with the number of stimuli (Fig. 14C). The sum of these functions produces the complex dependence of the total area bias shown in Fig. 14A. 


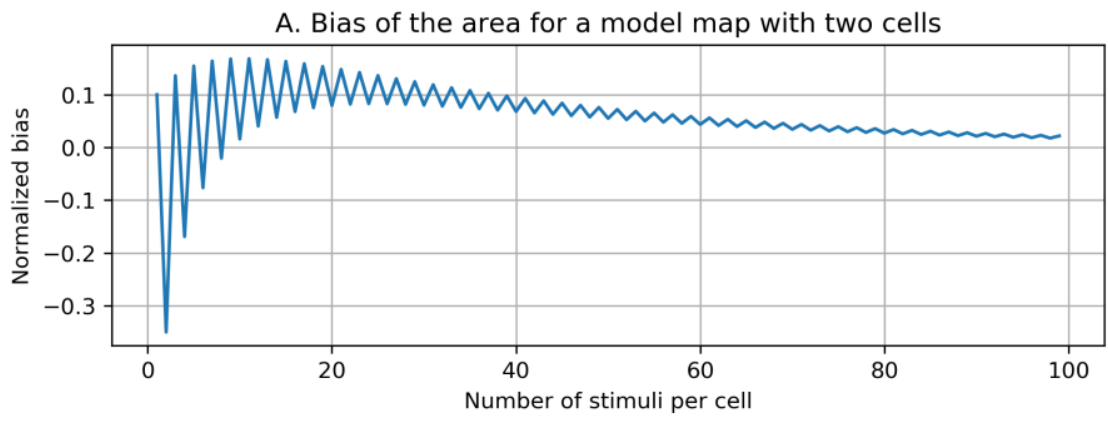

B. Mean contribution of the 1 st cell to the area, $p(M E P)=0.4$

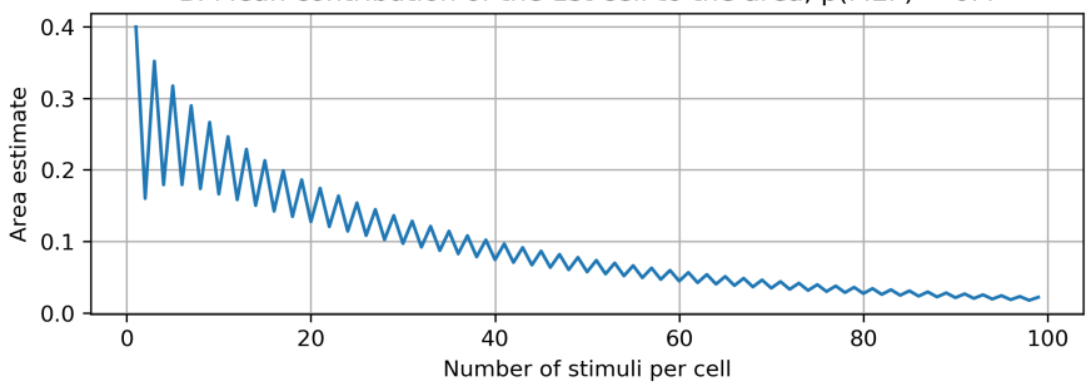

C. Mean contribution of the 2 nd cell to the area, $p(M E P)=0.7$

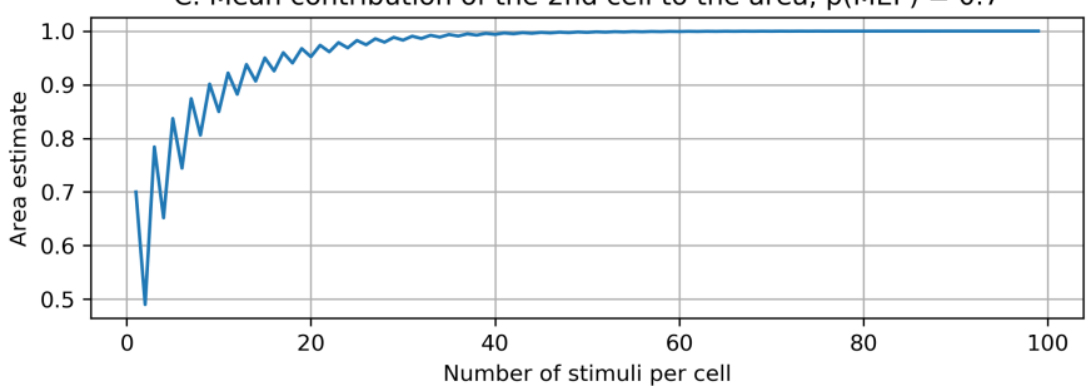

Figure 14. A. Non-monotonic dependence of the bias of $A_{\text {half }}$ (the area of the cells with more than half suprathreshold MEPs) on the number of stimuli per cell. The bias is calculated for a model muscle representation consisting of two grid cells with the probabilities of suprathreshold MEPs equal to 0.4 and 0.7. B. Contribution of the first cell to the estimate of $A_{\text {half. }}$ C. Contribution of the second cell to the estimate of $A_{\text {half. }}$

It is important to note that the decrease of the absolute value of the bias in Fig. 14A is slow. Reducing the bias to below 0.1 requires approximately 40 stimuli per cell, which is arguably impractical for real mapping with a considerable number of grid cells. The slow decrease is related to the presence of cells with probabilities of suprathreshold MEPs not far from the threshold probability ( 0.4 and 0.5 in the considered case). The TMS map visualizations in Fig. 2A indicate that the cells cover the whole span of MEP probability. Thus, the definitions of the representation area based on a threshold on the fraction of positive MEPs (such as $\mathrm{A}_{\text {half }}$ ) can generally produce considerable subject-dependent biases and should be used with caution, especially regarding the interpretation of group comparisons, as well as area estimates obtained with different numbers of stimuli per cell.

604 1. Rotenberg, A.; Horvath, J.C.; Pascual-Leone, A. Transcranial Magnetic Stimulation; Humana Press: New York, 2014; ISBN 978-1-4939-0878-3. 258. 
610 4. Tarapore, P.E.; Tate, M.C.; Findlay, A.M.; Honma, S.M.; Mizuiri, D.; Berger, M.S.; Nagarajan, S.S. Preoperative 611 multimodal motor mapping: a comparison of magnetoencephalography imaging, navigated transcranial magnetic 612 stimulation, and direct cortical stimulation. J. Neurosurg. 2012, 117, 354-362.

613 5. Picht, T.; Frey, D.; Thieme, S.; Kliesch, S.; Vajkoczy, P. Presurgical navigated TMS motor cortex mapping 614 improves outcome in glioblastoma surgery: a controlled observational study. J. Neurooncol. 2016, 126, 535-543.

615 6. Ziemann, U.; Ilić, T. V.; Jung, P. Chapter 3 Long-term potentiation (LTP)-like plasticity and learning in human motor cortex - investigations with transcranial magnetic stimulation (TMS). Suppl. Clin. Neurophysiol. 2006, 59, $19-25$. of cortical neuroplasticity associated with multidirectional novel motor skill training: A TMS mapping study. Exp. Brain Res. 2013. responses evoked by transcranial magnetic stimulation during the acquisition of new fine motor skills. $J$. Neurophysiol. 1995, 74, 1037-1045.

Raffin, E.; Siebner, H.R. Use-Dependent Plasticity in Human Primary Motor Hand Area: Synergistic Interplay Between Training and Immobilization. Cereb. Cortex 2019, 29, 356-371.

10. Lüdemann-Podubecká, J.; Nowak, D.A. Mapping cortical hand motor representation using TMS: A method to assess brain plasticity and a surrogate marker for recovery of function after stroke? Neurosci. Biobehav. Rev. 2016, 69, 239-251.

11. Schabrun, S.M.; Stinear, C.M.; Byblow, W.D.; Ridding, M.C. Normalizing Motor Cortex Representations in Focal Hand Dystonia. Cereb. Cortex 2009, 19, 1968-1977.

12. Freund, P.; Rothwell, J.; Craggs, M.; Thompson, A.J.; Bestmann, S. Corticomotor representation to a human forearm muscle changes following cervical spinal cord injury. Eur. J. Neurosci. 2011, 34, 1839-1846.

13. Cortes, M.; Thickbroom, G.W.; Elder, J.; Rykman, A.; Valls-Sole, J.; Pascual-Leone, A.; Edwards, D.J. The corticomotor projection to liminally-contractable forearm muscles in chronic spinal cord injury: a transcranial magnetic stimulation study. Spinal Cord 2017, 55, 362-366.

14. Chervyakov, A. V.; Bakulin, I.S.; Savitskaya, N.G.; Arkhipov, I. V.; Gavrilov, A. V.; Zakharova, M.N.; Piradov, M.A. Navigated transcranial magnetic stimulation in amyotrophic lateral sclerosis. Muscle Nerve 2015, 51, 125131.

639 15. Liepert, J.; Graef, S.; Uhde, I.; Leidner, O.; Weiller, C. Training-induced changes of motor cortex representations in stroke patients. Acta Neurol. Scand. 2000, 101, 321-6. 
versus functional MRI. Neuroimage 2013, 66, 531-542.

644 17. Kraus, D.; Gharabaghi, A. Neuromuscular Plasticity: Disentangling Stable and Variable Motor Maps in the Human Sensorimotor Cortex. Neural Plast. 2016, 2016.

646 18. Pellegrini, M.; Zoghi, M.; Jaberzadeh, S. The effect of transcranial magnetic stimulation test intensity on the amplitude, variability and reliability of motor evoked potentials. Brain Res. 2018, 1700, 190-198.

19. Julkunen, P. Methods for estimating cortical motor representation size and location in navigated transcranial magnetic stimulation. J. Neurosci. Methods 2014, 232, 125-133.

20. Classen, J.; Knorr, U.; Werhahn, K.J.; Schlaug, G.; Kunesch, E.; Cohen, L.G.; Seitz, R.J.; Benecke, R. Multimodal output mapping of human central motor representation on different spatial scales. J. Physiol. 1998, 512 ( Pt 1), $163-79$.

21. Kleim, J.A.; Kleim, E.D.; Cramer, S.C. Systematic assessment of training-induced changes in corticospinal output to hand using frameless stereotaxic transcranial magnetic stimulation. Nat. Protoc. 2007.

22. Uy, J.; Ridding, M.C.; Miles, T.S. Stability of maps of human motor cortex made with transcranial magnetic stimulation. Brain Topogr. 2002, 14, 293-297.

23. Corneal, S.F.; Butler, A.J.; Wolf, S.L. Intra- and intersubject reliability of abductor pollicis brevis muscle motor map characteristics with transcranial magnetic stimulation. Arch. Phys. Med. Rehabil. 2005, 86, 1670-1675.

24. McGregor, K.M.; Carpenter, H.; Kleim, E.; Sudhyadhom, A.; White, K.D.; Butler, A.J.; Kleim, J.; Crosson, B. Motor map reliability and aging: A TMS/fMRI study. Exp. Brain Res. 2012, 219, 97-106.

661 25. Ngomo, S.; Leonard, G.; Moffet, H.; Mercier, C. Comparison of transcranial magnetic stimulation measures obtained at rest and under active conditions and their reliability. J. Neurosci. Methods 2012, 205, 65-71.

26. Cavaleri, R.; Schabrun, S.M.; Chipchase, L.S. The number of stimuli required to reliably assess corticomotor excitability and primary motor cortical representations using transcranial magnetic stimulation (TMS): a systematic review and meta-analysis. Syst. Rev. 2017, 6, 48 .

27. Forster, M.-T.; Limbart, M.; Seifert, V.; Senft, C. Test-retest Reliability of Navigated Transcranial Magnetic Stimulation of the Motor Cortex. Oper. Neurosurg. 2014, 10, 51-56.

28. Schönfeldt-Lecuona, C.; Thielscher, A.; Freudenmann, R.W.; Kron, M.; Spitzer, M.; Herwig, U. Accuracy of stereotaxic positioning of transcranial magnetic stimulation. Brain Topogr. 2005, 17, 253-9.

29. Julkunen, P.; Säisänen, L.; Danner, N.; Niskanen, E.; Hukkanen, T.; Mervaala, E.; Könönen, M. Comparison of navigated and non-navigated transcranial magnetic stimulation for motor cortex mapping, motor threshold and motor evoked potentials. Neuroimage 2009, 44, 790-795. 
31. Opitz, A.; Zafar, N.; Bockermann, V.; Rohde, V.; Paulus, W. Validating computationally predicted TMS stimulation areas using direct electrical stimulation in patients with brain tumors near precentral regions. NeuroImage Clin. 2014, 4, 500-507.

32. Raffin, E.; Pellegrino, G.; Di Lazzaro, V.; Thielscher, A.; Siebner, H.R. Bringing transcranial mapping into shape: Sulcus-aligned mapping captures motor somatotopy in human primary motor hand area. Neuroimage 2015, 120, $164-175$.

33. Zrenner, C.; Desideri, D.; Belardinelli, P.; Ziemann, U. Real-time EEG-defined excitability states determine efficacy of TMS-induced plasticity in human motor cortex. Brain Stimul. 2018, 11, 374-389. cortex with magnetic stimulation: factors affecting accuracy and reproducibility. Electroencephalogr Clin Neurophysiol 1992, 85, 9-16.

35. Thickbroom, G.W.; Byrnes, M.L.; Mastaglia, F.L. A model of the effect of MEP amplitude variation on the accuracy of TMS mapping. Clin. Neurophysiol. 1999, 110, 941-943.

36. Van De Ruit, M.; Perenboom, M.J.L.; Grey, M.J. TMS brain mapping in less than two minutes. Brain Stimul. 2015, 8, 231-239.

37. Novikov, P.A.; Nazarova, M.A.; Nikulin, V. V TMSmap - Software for Quantitative Analysis of TMS Mapping

39. Oldfield, R.C. The assessment and analysis of handedness: The Edinburgh inventory. Neuropsychologia 1971, 9 , 97-113.

40. Jonker, Z.D.; Vliet, R. Van Der; Hauwert, C.M.; Gaiser, C.; Joke, H.M.; Geest, J.N. Van Der; Donchin, O.; Ribbers, G.M.; Frens, M.A.; Selles, R.W. TMS motor mapping: comparing the absolute reliability of digital reconstruction methods to the golden standard. Brain Stimul. 2018.

699 41. Efron, B. Bootstrap Methods: Another Look at the Jackknife. Ann. Stat. 1979, 7, 1-26.

$700 \quad$ 42. Hollander, M.; Wolfe, D.A. Nonparametric statistical methods; Wiley, 1999; ISBN 0471190454.

701 43. Gehan, E.A. A Generalized Wilcoxon Test for Comparing Arbitrarily Singly-Censored Samples. Biometrika 1965, 52, 203.

703 44. Flandin, G.; Friston, K. Statistical parametric mapping (SPM). Scholarpedia 2008, 3, 6232.

704 45. McGraw, K.O.; Wong, S.P. Forming inferences about some intraclass correlation coefficients. Psychol. Methods 1996, 1, 30-46. 
708 47. Cavaleri, R.; Schabrun, S.M.; Chipchase, L.S. The reliability and validity of rapid transcranial magnetic stimulation mapping. Brain Stimul. 2018, 1-5.

710 48. Mortifee, P.; Stewart, H.; Schulzer, M.; Eisen, a Reliability of transcranial magnetic stimulation for mapping the human motor cortex. Electroencephalogr. Clin. Neurophysiol. 1994, 93, 131-7.

712 